\title{
VERIFICATION OF A NOVEL METHOD OF DETECTING FAULTS IN MEDIUM-VOLTAGE SYSTEMS WITH COVERED CONDUCTORS
}

\author{
Stanislav Mišák ${ }^{1}$, Štefan Hamacek ${ }^{1)}$, Mikołaj Bartłomiejczyk ${ }^{2)}$ \\ 1) V̌̌B-Technical University of Ostrava, Faculty of Safety, Engineering, 17 Listopadu 15/2172, 70833 Ostrava-Poruba, Czech Republic \\ (stanislav.misak@vsb.cz,stefan.hamacek@seznam.cz) \\ 2) Gdańsk University of Technology, Faculty of Electrical and Control Engineering, G. Narutowicza 11/12, 80-233 Gdańsk, Poland \\ $\triangle$ mikolaj.bartlomiejczyk@pg.gda.pl,+48 58347 1416)
}

\begin{abstract}
This paper describes the use of new methods of detecting faults in medium-voltage overhead lines built of covered conductors. The methods mainly address such faults as falling of a conductor, contacting a conductor with a tree branch, or falling a tree branch across three phases of a medium-voltage conductor. These faults cannot be detected by current digital relay protection systems. Therefore, a new system that can detect the above mentioned faults was developed. After having tested its operation, the system has already been implemented to protect mediumvoltage overhead lines built of covered conductors.
\end{abstract}

Keywords: fault trees, partial discharges, fault diagnosis, power engineering, power overhead lines.

(C) 2017 Polish Academy of Sciences. All rights reserved

\section{Introduction}

In today's technologically developed times, great emphasis is put on providing a reliable supply of electrical energy to households and the transport, industrial and other sectors. New, advanced technologies and methods of timely fault detection help to faster remove of faults, thus increasing reliability of the electrical energy supplies. A new system of overhead lines with SAX-type covered conductors (hereinafter CCs) was developed in Finland in 1976 [1-3].

Such overhead lines were built in Norway and Sweden as well, and gradually appeared in other member states of the European Union. Employing CCs in the construction of these overhead lines is not much different from using bare wires in outdoor overhead lines; the only difference is in using XLPE insulation in the former [4-6]. By using the insulation, a fault rate of these overhead lines is reduced and it is possible to build the lines in places that are not easily accessible, e.g. in densely forested areas. Unlike typical outdoor overhead lines with AlFe conductors, when a tree branch falls on overhead lines with CCs, an immediate interphase shortcircuit does not occur; therefore, the risk of disconnection from the electrical energy supply is reduced $[7,8]$.

A disadvantage of operating overhead lines with CCs is the inability to detect a fault, e.g. in the case of falling of a conductor [9]. When this kind of fault occurs, there is no earth fault and the limit values of digital relay protection starting elements are not exceeded $[10,11]$. Hence, digital relay protection systems are not able to detect this type of fault. For such faults, as well as other types of faults associated with CCs, such as a tree branch falling on or merely contacting with a CC, partial discharges (hereinafter PDs) - inner, outer and surface PDs appear in the fault location (e.g. the point of contact between a conductor and the ground or between a tree branch and CC) [12]. These PDs gradually damage the insulation of the lines. The activity of PDs enlarges the area of a fault, and other insulated areas are being degraded. 
During this process, the conductor insulation breaks down and causes a fault (interphase or earth fault). The VŠB-TUO team developed a methodology based on the principle of finding a relation between the occurrence and characteristics of PDs and the origin of faults in overhead lines with CCs. Based on this methodology, a number of measurements were made. Using these measurements, procedures for detecting fault indicators in real medium-voltage overhead lines with CCs were gradually created. The effects of PDs can be worsened by other factors, such as humidity, temperature, solar radiation, atmospheric pressure, rainfall, aerosols, dust, sand, micro- and macro-organisms and the mechanical deterioration of conductors. Based on the information provided above, the occurrence of PDs is the best indicator of overhead line faults $[9,12,13]$.

The current worldwide research is focused on the development of methodologies for detecting the above mentioned faults in the operation of overhead lines with CCs. Most of these methodologies are based on evaluation of PD current pulses, and their selectivity and sensitivity depend on the measurement technique, which must meet high standards, especially the ability to detect pulse sources, and which must include a high-resolution A/D converter and efficient hardware for data processing [14-16].

The system developed by the team at VŠB-Technical University of Ostrava in the Czech Republic (hereinafter VŠB-TUO) and presented in this study, is able to specify the location and type of a fault $[17,18]$. The system operation is based on analysing PD voltage signal pulses using suggested indicators of faults [9] and is not technically demanding.

\section{Method's principles}

The method developed by the VŠB-TUO team is based on finding a relation between the occurrence and characteristics of PDs and the origin of faults in overhead lines with CCs. As was stated above, in the first part, the occurrence of PDs is characterized by damaging the insulation system of a variety of devices. These PDs arise in areas of small cavities in the insulation system as an effect of extreme local electrical tension. A simple model and substitution diagram of simulating the voltage and current conditions inside the insulation system is shown in Fig. 1.

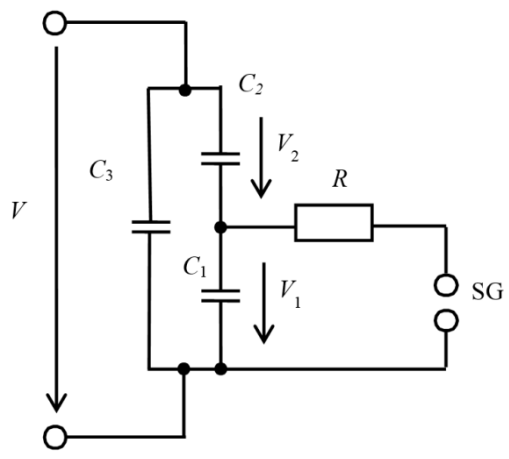

Fig. 1. A simple model of an insulation system with a cavity [19].

A cavity capacitance (the damaged part of the insulation) is represented by $C_{1}, C_{2}$ is a capacitance of the residual part of "healthy" insulation (a serial connection of $C_{2}$ and $C_{1}$ ), and $C_{3}$ presents a significant capacitance of other parts of insulation. A sphere gap (SG) represents flashover in the cavity area and $R$ is a resistivity of the discharge puncture after flashover on SG. When the insulation system is damaged and cavities inside it exist, the voltage on the cavity varies in time according to the formula: 


$$
v_{10}(t)=\frac{C_{2}}{C_{1}+C_{2}} v(t),
$$

where $v(t)$ is the power supply voltage. When the value of voltage on the cavity equals the voltage ignition value, a PD occurs. These frequent PDs are superposed onto the carrier of capacitive current component.

\subsection{Pulse component measurements}

The basic part of capacitive current is caused by the supply voltage and for evaluation of PD activity it is not relevant. However, PDs superposed onto the carrier capacitive current, named a pulse component, are very important for evaluation of PD activity inside the insulation system.

Two basic methods can be used for indirect evaluation of PD activity:

i) evaluation of $\mathrm{PD}$ activity in the insulation system of $\mathrm{CC}$ by measuring the current signal in CC by a Rogowski sensor [2], and

ii) evaluation of $\mathrm{PD}$ activity in the insulation system of $\mathrm{CC}$ by measuring the voltage signal of electrical stray field along the CC.

Both methods use for evaluation of PD activity a pulse component. The pulse component is generated by PD activity in the insulation system and its occurrence is characterized by a frequency domain of hundreds of $\mathrm{kH}$ to $\mathrm{MHz}$. The first method evaluates the pulse component generated by PD activity from the current measured by a Rogowski sensor. The main advantage of this method is a high selectivity of evaluation of PD activity. However, this high selectivity of PD evaluation requires both high sensitivity and accuracy of the Rogowski sensor over a wide frequency range because the pulse component is low-energetic and the measurement period is in the order of microseconds. These high requirements on the Rogowski sensor increase a price of the measurement chain, which is a great disadvantage of this method (i) [2].

The aim of this paper is to show the possibility of using the second method that evaluates the pulse component generated by PD activity as a voltage signal of the electrical stray field measured along an insulation system, in this case the insulation system of CC. The requirements for development of this method were: high selectivity of fault detection, high reliability and a low price of the prototype $\mathrm{CC}$ fault detector.

As was stated in the previous section, the pulse component generated by PD activity in an insulation system of CC is measured as the voltage signal of electrical stray field along the CC. This voltage signal was measured by a capacitor voltage divider (CA-CB) from a circular metal sensor (a circular coil; see $C_{\text {coil }}$ in Fig. 2) through its coupling capacitor $C_{\text {coup }}$ to the CC. As a sensor it is possible to use, for example, an inductor wound on the $\mathrm{CC}$ surface, where $C_{\text {coup }}$ will be given by the number of turns (see Fig. 2).

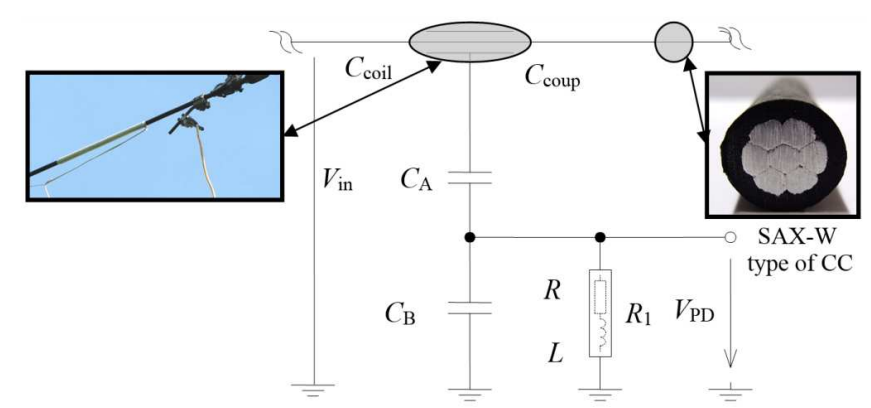

Fig. 2. The principle of measuring the pulse component of voltage signal including an analogue low-pass filter. 
The time characteristic of voltage signal of the electrical stray field on the CC surface is then amplitude-conditioned by means of a capacitor voltage divider with a fixed dividing ratio given by the capacities $C_{\mathrm{A}}$ and $C_{\mathrm{B}}$; see Fig. 2 . When the capacitor voltage divider is loaded via a properly selected resistor $R_{1}$, which may be additionally equipped with a self-inductance, a low pass with advantageous amplitude characteristic occurs; see Fig. 3 (the vertical axis a damping characteristic in $\mathrm{dB}$, the horizontal axis - a frequency in $\mathrm{Hz}$ ).

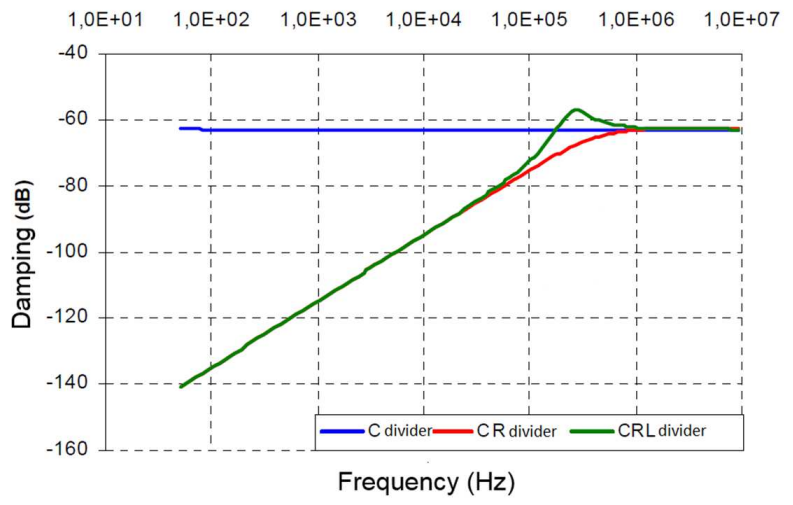

Fig. 3. Frequency characteristics of a coupling circuit in $\mathrm{CC}$ for various endings of the capacitor voltage divider ( $C$-divider, $C$-divider with $R, C$-divider with $R L$ ).

The voltage signal of the electrical stray field measured by the sensor as a function of time is shown in Fig. 4, where the red line defines the output voltage signal using a C-divider and the green line defines the output voltage signal using a C-divider with a resistor $R_{1}$ connected in parallel. From the green line in Fig. 4, the required pulse component of voltage signal generated by PD activity is visible. This signal can be filtered using a digital filter for elimination of the carrier frequency of power supply and for further specification of a typical frequency range for PD activity (mostly $1-10 \mathrm{MHz}$ ). In this way, the original pattern of PD (hereinafter the PD-pattern) arises, corresponding to the actual state of CC insulation system. Next, detection of a CC fault is possible by evaluating this PD-pattern. It means that the shape of the PD-pattern shows the actual state of CC insulation system. For evaluation of the PDpattern it is possible to use some indicators in the time and frequency domain, whereas the requirements for selection of the indicators are: (i) a short evaluation time; (ii) low memory requirements and (iii) selective evaluation.

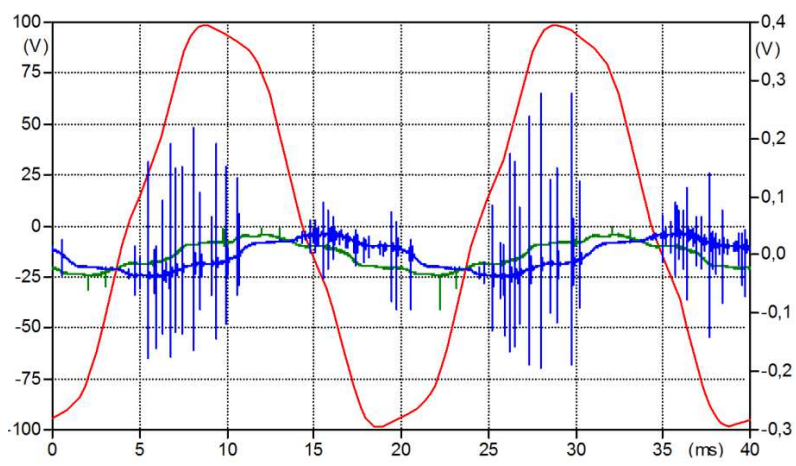

Fig. 4. The functions of output voltage signal vs time using: a C-divider (red line), a C-divider with a resistor $R_{\mathrm{I}}$ connected in parallel for the no-fault state (green line), a C-divider with a resistor $R_{1}$ connected in parallel for a fault state (blue line). 
So, for example, calculations of the True Root Mean Square value (hereinafter the TRMS) of the PD-pattern and the mean value of the PD-pattern or the frequency $\left(\mathrm{s}^{-1}\right)$ of PDs in the PD-pattern (hereinafter $n$ ) for the first time were verified as indicators in evaluation of the actual state of CC insulation by the research team at VŠB-TU Ostrava. A function of the TRMS of PDpattern (hereinafter VTRMS) vs time is approximately zero in the case of fault-free operation, because the PD activity is irrelevant (see the green line in Fig. 4). If a fault in the CC insulation exists, the PD activity is relevant and the TRMS value is increased; see the blue line in Fig. 4.

The next modification of the PD-pattern is extraction of a low-level PD signal from the background of an interfering signal source, which is composed of signal sources from the external environment (e.g. radio transmitters).

Therefore, to modify the electrical stray field voltage signal, we used an IIR Butterworth digital filter with an infinite pulse response and maximally flat permeable and impermeable ranges.

Based on the selected basic measurement system for recording and modifying the signal, we developed a fault-detecting system able to analyse the processed data, which is described in the following section.

\section{Pulse component measurements}

The main requirement for a fault-detecting system is the oscilloscopic measurement of varying the PD-pattern voltage of electrical stray fields around medium-voltage overhead lines with CCs. We used a measuring card connected via a PCI interface for this purpose. In addition to measuring the PD-pattern, the fault detector measures other conditions, such as temperature, pressure, humidity and global radiation (exposure), since the discharge activity is affected by these factors. These conditions affect, for example, the frequency of PD occurrence, the PD amplitude shape and size .

All measured and processed data are sent by the GSM network to an external computer, where they are processed and an overhead line fault condition is analysed. If the threshold values of indicators are exceeded, the fault detector sends a warning signal to the CC operator.

The fault detector also includes additional control electronic circuits which are used to switch the PC to automatic control, and to measure temperature inside the switchboard (in the case of unnecessary heating). The device is powered by a battery which is charged by photovoltaic panels. Thus, it is possible to use the detector anytime on overhead lines with CCs, even when there is no possibility to get energy from the distributed system power supply.

\section{Measurement of real medium-voltage overhead lines with CCs}

\subsection{Verification of fault detector function}

The CC fault detector prototype was installed on 22-kV overhead lines with CCs (Fig. 5). The main aim was to verify the effect of CC network topology on sensitivity and selectivity of the fault detector prototype and its long-term application in a chosen location with mediumvoltage overhead lines.

To confirm the conclusions and to assess the fault detector functionality, experimental measurements were performed on real overhead lines with CCs $(22 \mathrm{kV})$ in December 2012 and February 2013. The aim of measurements was to obtain new data and to verify the results already gained during the previous period, particularly setting the frequency threshold values $\left(n=100 \mathrm{~s}^{-1}\right)$ and the voltage level of the generated PD voltage signal pulse component rootmean-square $\left(V_{\text {TRMS }}=39 \mathrm{mV}\right)$. The indicator $n\left(\mathrm{~s}^{-1}\right)$ is a frequency of PDs' activity and, as well as $V_{\text {TRMS }}$, was analysed during experimental measurements in the laboratory conditions $[9,13]$. 
When these values are exceeded, the fault detector sends a report about a fault in the overhead lines. Different types of faults were simulated on overhead lines with CCs, particularly faults that cannot be detected by current digital relay protection systems. In particular, selectivity and sensitivity of both the methodology and fault detector were verified for such faults as a contact between a tree branch and a phase conductor, a conductor falling on the ground and a contact between two phase conductors. Due to the insulation of conductors, these faults do not have to be cleared immediately. However, the tested fault detector can detect these faults before degradation factors destroy integrity of the insulation in the fault location and cause a single phase-to-earth short-circuit.

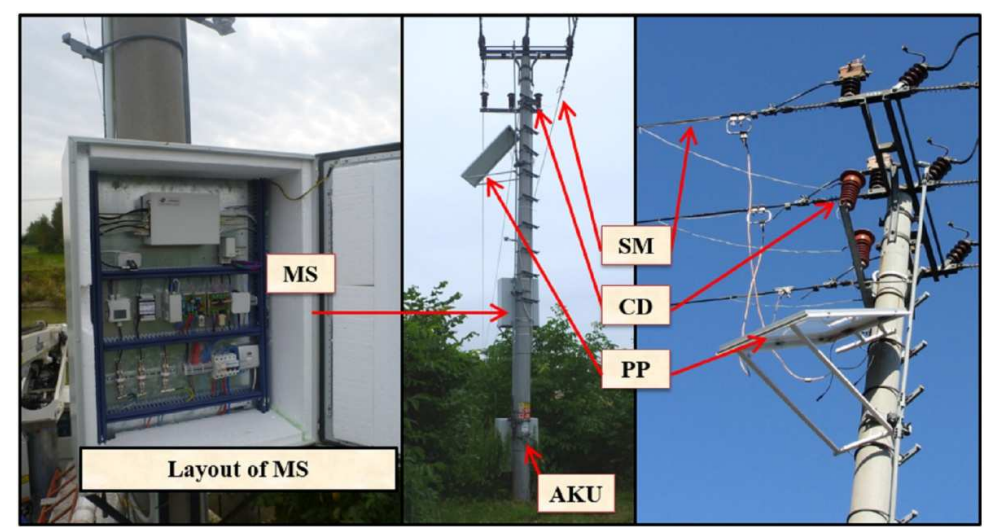

Fig. 5. Arrangement of the fault detector on a post of medium-voltage overhead lines with CCs [21]:

MS - Measuring System, BAT - Battery, SM - Sensing Member, CD - C-Divider, PP - Photovoltaic Panel.

The results of these experimental measurements are presented below:

Fault no. 1 - the point of contact between a tree and CC;

Fault no. 2 - breaking off of a CC and its subsequent falling on the ground.

To compare the faulty and fault-free conditions (Fig. 6), we had to measure the PD-pattern in the operating conditions. From the PD-pattern (Fig. 6), the existence of a pulse component is evident, although the overhead lines with CCs were free of fault generated by a corona discharge at the connector endings on towers with CCs and also by modulation of interfering signals (e.g. radio waves). The software component of fault detection system can recognize the interference and evaluate the signal as fault-free.

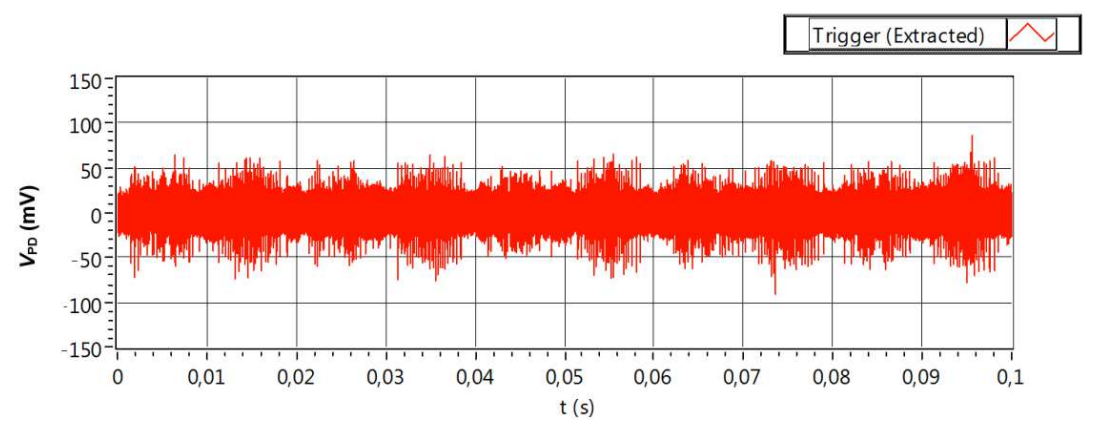

Fig. 6. The $V_{\text {TRMS }}$ of PD-pattern of CC for the no-fault state. 


\section{Fault no. 1 - point of contact between tree and phase conductor.}

The first measurement was simulation of the point of contact with a phase conductor. This fault was simulated by considering a tree branch located near the outer phase of CC (Fig. 7a). This type is the worst detectable fault because a contact between a tree and a conductor can, in real situations, be a result of transitory conditions, e.g. wind. Another fact contributing to the difficulty in detecting this type of fault is the minimal area of conductor damage and the corresponding minimal occurrence of PDs in the fault location. By further analysing the pulse component of the generated PD-pattern in the fault location, we determined the following indicator values: $n=272 \mathrm{~s}^{-1}$; $V_{\text {TRMS }}=68 \mathrm{mV}$.

Considering the exceeded indicator threshold values, selectivity and sensitivity of the methodology as well as the fault detector functionality were verified. Changing of the measured signal in time, shown below (Fig. 7b), clearly indicates that over a period of $0.06 \mathrm{~s}$ no PD occurred. This lack of PD was caused by an imperfect connection between a tree branch and CC. The fault detector evaluates faults continually; therefore, this transient effect on the general selectivity of fault detector is eliminated.

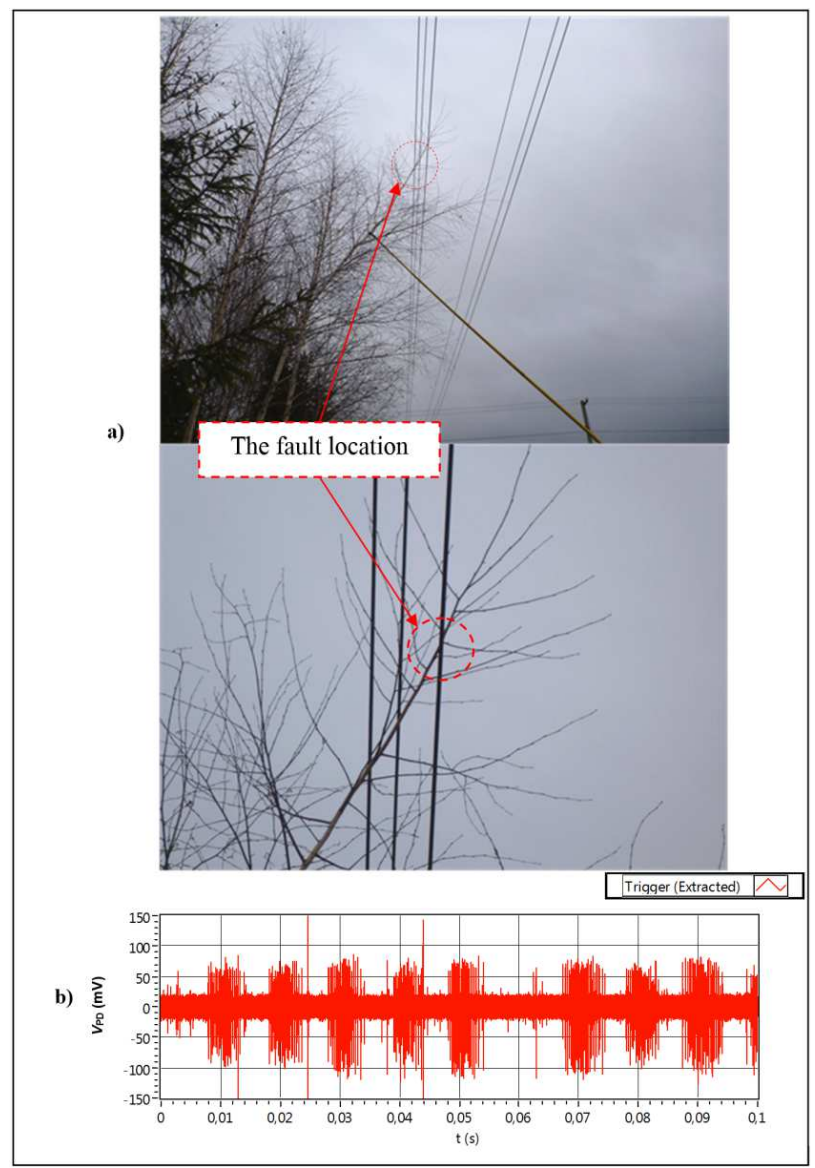

Fig. 7. Fault no. 1 - a contact between a tree branch and overhead lines with CC (a); the change of PD-pattern in time, fault no. 1 (b). 


\section{Fault no. 2 - breaking off of $\mathrm{CC}$ and its subsequent falling on ground.}

The second simulated fault was the breaking off of a CC and the conductor's subsequent falling on the ground. The fault was simulated by connecting an SAX-W-type conductor (22$\mathrm{kV}$ ) to the overhead lines. The CC end was then placed down on the ground (Fig. 8a). At the point of contact between the conductor and the ground, PDs evolve and the voltage signal, which afterwards travels along the insulation of the conductor as a wave, is recorded by the fault detector. This type of fault is easily detected; therefore, in the step the conductor was placed on the ground covered with grass. The contact with the ground was partly weakened by grass. Fig. $8 \mathrm{~b}$ shows the PD-pattern recorded after using the IIR Butterworth filter; the measured values were: $n=174 \mathrm{~s}^{-1} ; V_{\text {TRMS }}=68 \mathrm{mV}$.

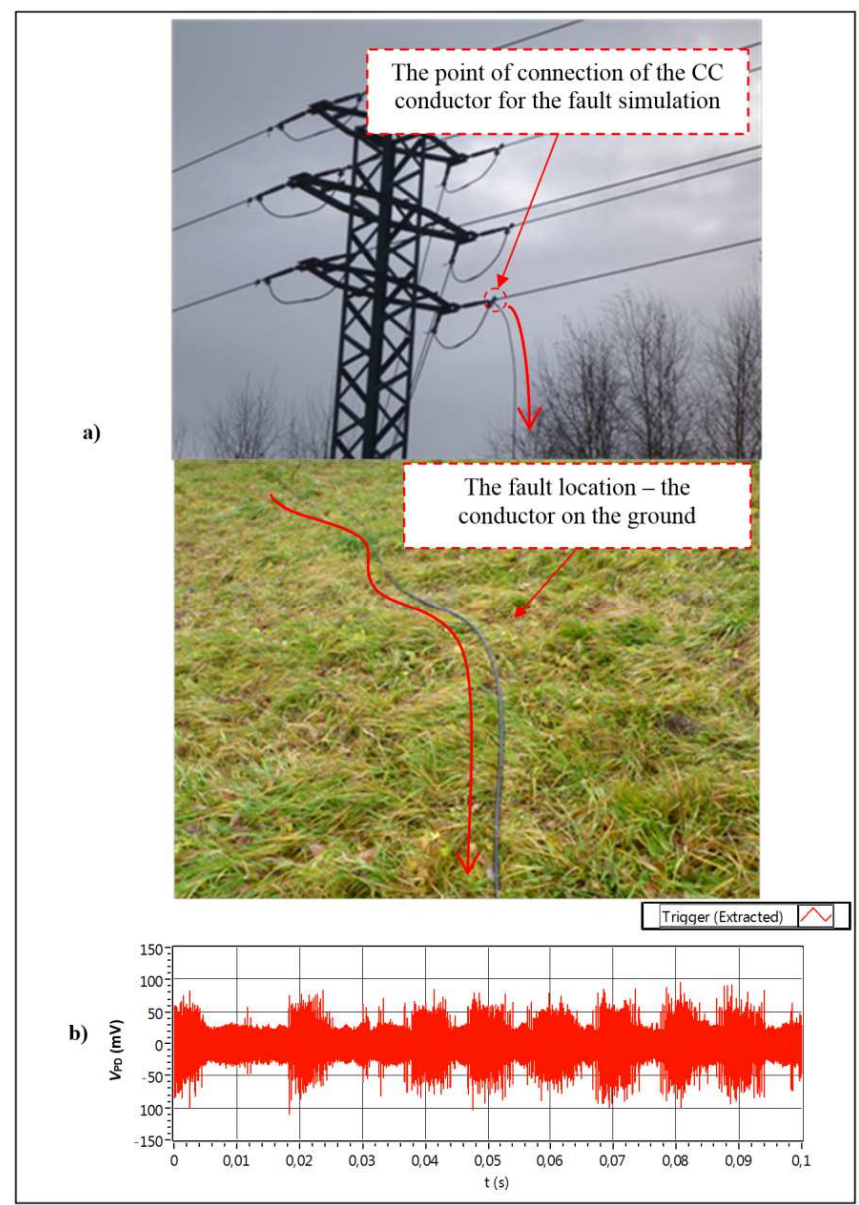

Fig. 8. Fault no. 2 - falling of a CC on the ground (a); the PD-pattern, fault no. 2 (b).

To provide a general summary of the measured values, we created a table of climatic conditions (Table 1) and a table of chosen fault indicators (Table 2). As shown in Table 1, the measurements were performed in unfavourable weather conditions. Thus, if the temperature was approximately equal to zero and humidity was high, we presumed the occurrence of PD in overhead lines in the form of a corona. Coronas frequently occur in areas of high local electrical gradients, where inhomogeneous electrical fields are generated, such as sharp edges, bushings, connectors and other construction parts of overhead lines. Table 2 shows that the highest 
frequency values $n$ occurred during a contact between a tree branch and a phase conductor (fault no. 1). The high values were caused mainly by the fact that tree bark was soaked with water; thus, when the conductivity increased, the PD activity increased as well. When the conductor fell on the ground, we presumed that the highest energy of the PD voltage pulses occurred; thus, the $V_{\text {TRMS }}$ value was also the highest of all the measured values in this case.

Table 1. The measured values of climatic conditions.

\begin{tabular}{|c|c|}
\hline Temperature of the surroundings $\left({ }^{\circ} \mathrm{C}\right)$ & 3,2 \\
\hline Humidity $(\%)$ & 85,1 \\
\hline Dew point $\left({ }^{\circ} \mathrm{C}\right)$ & 1 \\
\hline Atmospheric pressure $(\mathrm{hPa})$ & 968.5 \\
\hline Global radiation $\left(\mathrm{W} \cdot \mathrm{m}^{-2}\right)$ & 0 \\
\hline${\text { Temperature in switchboard }\left({ }^{\circ} \mathrm{C}\right)}$ & 5,4 \\
\hline
\end{tabular}

Table 2 . The values of frequency and $V_{\text {TRMS. }}$

\begin{tabular}{|c|c|c|}
\hline & $\boldsymbol{V}_{\text {TRMS }}(\mathrm{mV})$ & $\boldsymbol{n}\left(\mathbf{s}^{-1}\right)$ \\
\hline Operating condition & 9 & 17 \\
\hline Fault no. 1 & 45 & 272 \\
\hline Fault no. 2 & 68 & 174 \\
\hline
\end{tabular}

\subsection{Long-term measurement of medium-voltage (22 kV) overhead lines with CCs}

After long-term experimental measurements, the fault detector was put into operation on MV $(22 \mathrm{kV})$ overhead lines with CCs. During its operation in May and October, the fault detector sent to the local $\mathrm{CC}$ overhead lines' operator a warning report indicating that the threshold values of indicators had been exceeded. In particular, the following values were measured by the detector: $n=150 \mathrm{~s}^{-1}(150>100)$ and $V_{\text {TRMS }}=42 \mathrm{mV}(42>39)$ of PD-pattern. At first, during May, the breakdown service of distribution system operator made a round along MV overhead lines with CCs and detected a fault in a site at a $3 \mathrm{~km}$ distance from the detector. The breakdown service detected falling of a tree branch across three phases.

The observed values ( $V_{\text {TRMS }}$ and $n$ ) were analysed step by step every day and the mean value of these data was evaluated at the end of individual month. Next, the tangent of these values was evaluated, too. The graphical output of this analysis for $V_{\text {TRMS }}$ and $n$ can be seen in Fig. 9 .

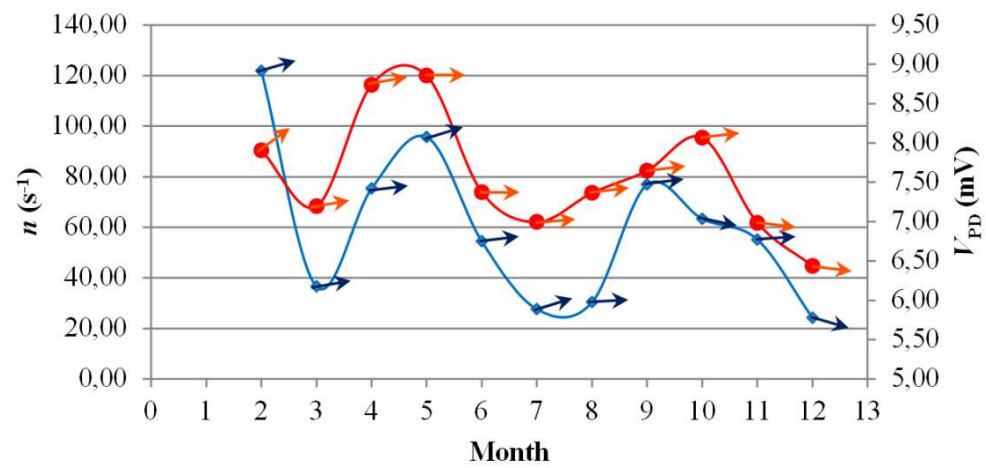

Fig. 9. Mean values of $n$ and $V_{\text {TRMS }}$ of PD-pattern for individual months of the year. 
From these graphical outputs a decrease in PD activity after elimination of the fault is evident until July. However, the second warning report was sent by the detector during October, when the threshold values ( $V_{\text {TRMS }}$ and $n$ of PD-pattern) were exceeded again. The breakdown service of distribution system operator made a round along MV overhead lines with CCs again. However, no fault in CCs has been found. Nevertheless, the detector sent the warning report because the threshold values ( $V_{\text {TRMS }}$ and $n$ of PD-pattern) were exceeded. The PD-pattern from the detector is shown in Fig. $10 \mathrm{~b}$ (red line - left axis).

Therefore, the breakdown service scanned the overhead lines with CCs in more detail using a corona camera and detected a fault in the same place as the previous fault. A photo illustrating the fault is presented in Fig. 10a. From this photo there is visible a damage on the CC surface. This damage was caused by the PD activity of the previous fault when a tree branch fell across three phases. This fault was cleared by the breakdown service during May. However, the insulation system of $\mathrm{CC}$ at the contact point of a tree branch with the insulation system had already been degraded. This degradation of the insulation system stopped during the summer months when high temperatures in the surroundings of CC caused "softening" of the insulation system and these small ruptures in the insulation system were eliminated. The opposite effect was caused during the autumn and winter months when the insulation system dried out and became more brittle as a result of low temperatures and low values of relative humidity. Therefore, the PD activity increased and degradation of the insulation system increased. The damaged line with $\mathrm{CC}$ was changed and - to prevent another similar accident - the trees in the area occupied by the MV overhead lines were trimmed. As a result, the PD activity (see Fig. 10b - green line - right axis) and monitored values ( $V_{\text {TRMS }}$ and $n$ of PD-pattern) decreased immediately (see Fig. 9) after executing these corrective and preventive steps.

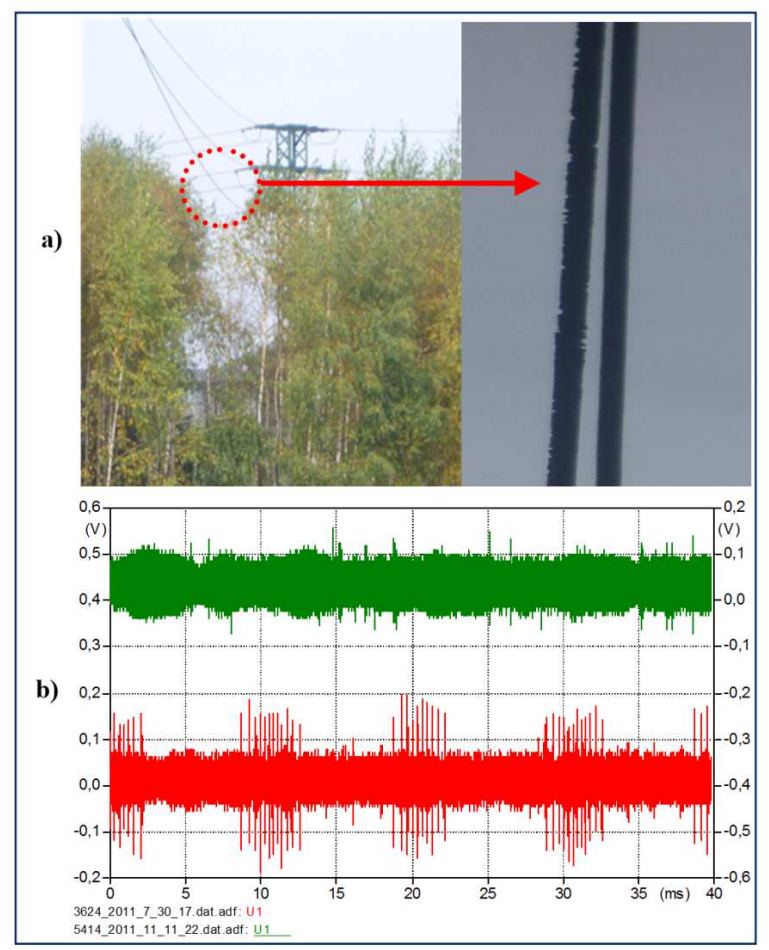

Fig. 10. The details of damage on a conductor in the measured location (a); the PD-pattern from the detector after it sent a warning report (left axis - red line) and the PD-pattern from the detector after carrying out the corrective and preventive steps (right axis - green line) (b). 


\section{Conclusion}

A system for detecting faults in overhead lines with CCs was designed based on a methodology developed by the research team at VŠB-Technical University of Ostrava. Prior to the construction of the fault detector, a number of experimental measurements $[7,8]$ and mathematical calculations using non-linear multi-physical programs (Lab View, ATP-DRAW, ANSYS) were carried out. Based on the results of these measurements and mathematical simulations, a fault detector prototype was constructed. The detector's functionality was tested by the long-term measurement of real MV overhead lines with CCs. During the measurement process, the performance of particular CC fault indicators defined in the research part of the project was gradually verified. Using these results, the evaluation component of calculating algorithm of the fault detector prototype was optimized. The fault detector was powered by storage batteries recharged by solar panels.

Based on the test results obtained for the fault detector by long-term measurements in real conditions, the ability of the detector to operate in the autonomous mode was successfully verified. The indicator threshold values applied to a signal on occurring a fault in MV overhead lines with CCs were specified. The fault detector is able to specify faults based on the measured threshold frequency values and the True Root Mean Square value of the electrical stray field voltage signal around the conductor. The fault detector recognizes faults in the overhead lines and sends a signal informing about the faults to the central computer. Further confirmation of the fault detector's ability to detect faults was obtained when the fault detector was put into operation. When the overhead lines were checked, it was observed that the cause of the detected fault was a tree branch that had fallen across all three phases of the lines. The fault caused partial damage to the conductor insulation, but due to the timely warning, there was no interphase short-circuit. Thus, the faults could be cleared within a short time, and more serious damage that could possibly cut the power supply to consumers could be prevented. Moreover, the repair costs were reduced.

In this study, we not only achieved the project objectives of producing a fault detector prototype and verifying its performance, but we also gathered a significant amount of information about the discharge activity of various insulation systems in various climatic conditions. This information can be used in developing other methods of protecting CCs as well as all other types of outdoor overhead lines and cable lines, and even insulation systems of other devices. In the future research, we aim to enhance the measurement quality of the fault detector, so that it is able to specify the location and type of faults that are detected.

\section{Acknowledgments}

This study was carried out within the framework of the IT4 Innovations Centre of Excellence project, reg. no. CZ.1.05/1.1.00/02.0070, supported by the Operational Programme "Research and Development for Innovations" funded by Structural Funds of the European Union and the state budget of the Czech Republic and the project Opportunity for Young Researchers, reg. no. CZ.1.07/2.3.00/30.0016, supported by the Operational Programme Education for Competitiveness and co-financed by the European Social Fund and the state budget of the Czech Republic and project reg. no. SP2014/49.

\section{References}

[1] Agarwal, H.K., Mukherjee, K., Barna, P. (2014). Partially and fully insulated conductor systems for low and medium voltage overhead distribution lines. Condition Assessment Techniques in Electrical Systems (CATCON). IEEE 1st International Conference, 104 (6-8), 100-104. 
[2] Hemmati, E., Shahrtash, S.M. (2012). Evaluation of shielded Rogowski coil for measuring partial discharge signals. Environment and Electrical Engineering (EEEIC), 11th International Conference, 446-450.

[3] Hemmati, E., Shahrtash, S.M. (2012). Evaluation of unshielded Rogowski coil for measuring partial discharge signals. Environment and Electrical Engineering (EEEIC), 11th International Conference, 434-439.

[4] Samimi, M.H., Mahari, A., Farahnakian, M.A., Mohseni, H. (2014). A Review on the Rogowski Coil Principles and Applications. Sensors Journal, IEEE, (99), 1, 1.

[5] Shafiq, M., Kutt, L., Lehtonen, M., Nieminen, T., Hashmi, M. (2013). Parameters Identification and Modeling of High-Frequency Current Transducer for Partial Discharge Measurements. Sensors Journal, IEEE, 13(3), 1081-1091.

[6] Nobrega, A.M., Martinez, M. L. B., de Queiroz, A.A.A. (2104). Analysis of the XLPE Insulation of Distribution Covered Conductors in Brazil. Journal of Materials Engineering and Performance, 23, $723-735$.

[7] Misak, S., Hamacek, S. (2010). Utilization of the Finite Element Method For Optimizing of Overhead Covered Conductors. Conference DAAAM 2010, Austria, Vienna.

[8] Makhkamova, I., Taylor, P.C., Bumby, J.R., Mahkamov, K. (2208). CFD analysis of the thermal state of an overhead line conductor. Universities Power Engineering Conference, UPEC 2008. 43rd International, 1-4.

[9] Hamacek, S. (2012). Problems of Covered Conductors Running. PhD Thesis. VŠB-TUO: VŠB-Technical University of Ostrava.

[10] Hashmi, G.M., Lehtonen, M., Nordman, M. (2010). Modeling and Experimental Verification of On-line PD Detection in MV Covered-conductor Overhead Networks. IEEE Trans. Dielectr. Electr. Insul., 17, 167-180.

[11] Hashmi, G.M., Lehtonen, M., Ametani, A. (2010). Modeling and Experimental Verification of Coveredconductor for PD Detection in Overhead Distribution Networks. IEEJ Trans. Power Energy, 130(7), Sec. B, 670-678.

[12] Misak, S., Hamacek, S., Bilík, P., Horinek, M., Petvaldsky, P. (2011). Problems associated with covered conductor fault detection. Electrical Power Quality and Utilisation (EPQU), 2011 11th International Conference, $1-5$.

[13] Hashmi, G.M., Lehtonen, M., Nordman, M. (2011). Calibration of on-line partial discharge measuring system using Rogowski coil in covered-conductor overhead distribution networks. Science, Measurement \& Technology, IET, 5(1), 5-13.

[14] Hashmi, G.M., Lehtonen, M. (2010). Effects of Rogowski Coil and Covered-Conductor Parameters on the Performance of PD Measurements in Overhead Distribution Networks. Int. J. Innovations Energy Syst. Power, 4(2), 14-20.

[15] Sudha, G., Valluvan, K.R. (2014). A novel approach to fault diagnosis of transmission line with Rogowski coil. International Review of Electrical Engineering, 9(3), 656-662.

[16] Isa, M., Elkalashy, N.I., Lehtonen, M., Hashmi, G.M., Elmusrati, M.S. (2012). Multi-end correlation-based PD location technique for medium voltage covered-conductor lines. IEEE Transactions on Dielectrics and Electrical Insulation, 19(3), 6215097, 936-946.

[17] Misak, S., Pokorny, V. (2015).Testing of a covered conductor's fault detectors. IEEE Transactions on Power Delivery, 30(3), 6957620, 1096-1103.

[18] Hamacek, S., Misak, S. (2013). Fault indicators of partial discharges in medium-voltage systems. Advances in Electrical and Electronic Engineering, 11(4), 284-289.

[19] Záliš, K. (2005). Částečné výboje v izolačních systémech elektrických strojů. 1st. ed. Praha: Academia. 